\title{
Spontaneous migration of a congenital intratympanic membrane cholesteatoma
}

\author{
Tae Hoon Kim, Kyu-Yup Lee, Da Jung Jung \\ Department of Otorhinolaryngology-Head and Neck Surgery, Kyungpook National University School of Medicine, Daegu, Korea
}

Congenital intratympanic membrane cholesteatoma (ITMC) is a rare type of congenital cholesteatoma located within the tympanic membrane. This lesion tends to increase in size over time. The development of ITMC can cause several complications such as hearing impairment, dizziness, facial palsy, and intracranial complications, similar to any other cholesteatoma. The treatment of congenital cholesteatoma requires the removal of the lesion through surgery, because disease progression induces bony destruction of the nearby tissue. Most patients presenting with this cholesteatoma type are also treated with primary surgical removal. However, we recently experienced a case of an ITMC that showed a natural transition to an external auditory canal cholesteatoma.

Keywords: Congenital cholesteatoma; External auditory canal; Tympanic membrane

\section{INTRODUCTION}

Congenital cholesteatoma is composed of a white mass located in the middle ear cavity and medial to the normal tympanic membrane. This condition can be diagnosed in the absence of otorrhea, tympanic membrane perforation, and a history of otologic surgery [1]. In patients with cholesteatomas, a growth of keratinized squamous epithelium is present that induces the bony destruction of nearby tissue. This bony destruction leads to hearing impairment, dizziness, facial palsy, and intracranial complications [2]. Therefore, the main purpose of cholesteatoma treatment is to surgically remove the lesion and preserve the patient's hearing. Congenital cholesteatoma located within the tympanic membrane is referred to

Received: May 30, 2018, Revised: July 5, 2018 Accepted: July 6, 2018

Corresponding Author: Da Jung Jung, Department of Otorhinolaryngology-Head and Neck Surgery, Kyungpook National University School of Medicine, 130, Dongdeok-ro, Jung-gu, Daegu 41944, Korea

Tel: +82-53-420-5777, Fax: +82-53-423-4524

E-mail: wjddk0731@naver.com as congenital intratympanic membrane cholesteatoma (ITMC). Its progression can also cause several complications, similar to any other cholesteatoma. In most case reports, the treatment is surgical removal [3-10]. We recently observed a case of ITMC in a child, wherein we originally planned to execute surgical treatment. However, this case developed into an external auditory canal cholesteatoma after 2 months. In this report, we describe this unusual case.

\section{CASE}

A 16-month-old female patient with a history of frequent otitis media visited our department. She had no history of otologic surgery. Otoscopic examination showed that her right tympanic membrane and right external auditory canal were normal, but there was a pearly white mass located at the end of the malleus handle, in the center of her left tympanic membrane (Fig. 1). A presumed diagnosis of an ITMC was made empirically. The patient did not have hearing impairment or any other signs of ear symptoms. Tympanometry showed a type A pattern for both ears (Fig. 2). Temporal bone compu-

Copyright $(52018$ Yeungnam University College of Medicine

This is an Open Access article distributed under the terms of the Creative Commons Attribution Non-Commercial License (http://creativecommons.org/licenses/by-nc/4.0/) which permits unrestricted non-commercial use, distribution, and reproduction in any medium, provided the original work is properly cited. 
ted tomography was performed, and the soft tissue density of a round mass was noted between the left tympanic membrane and the handle of the left malleus (Fig. 3). There were no other abnormal features of the inner structures or ossicles. At first, we planned surgery to remove the cholesteatoma. However, at follow-up consultation after 2 months, the mass was noted to have moved laterally into the external auditory canal (Fig. 4). The mass was easily removed with a hook without anesthesia. Histopathologic examination showed stratified

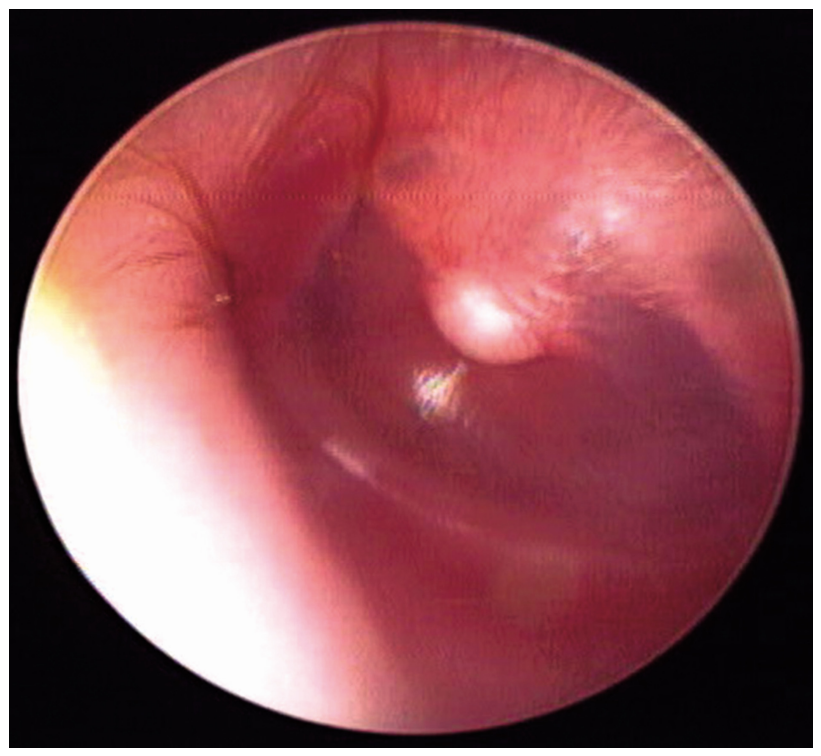

Fig. 1. Otoscopic finding. A pearly white mass is located at the end of handle of the left malleus, in the center of the left tympanic membrane.

Tymp: Sweep right

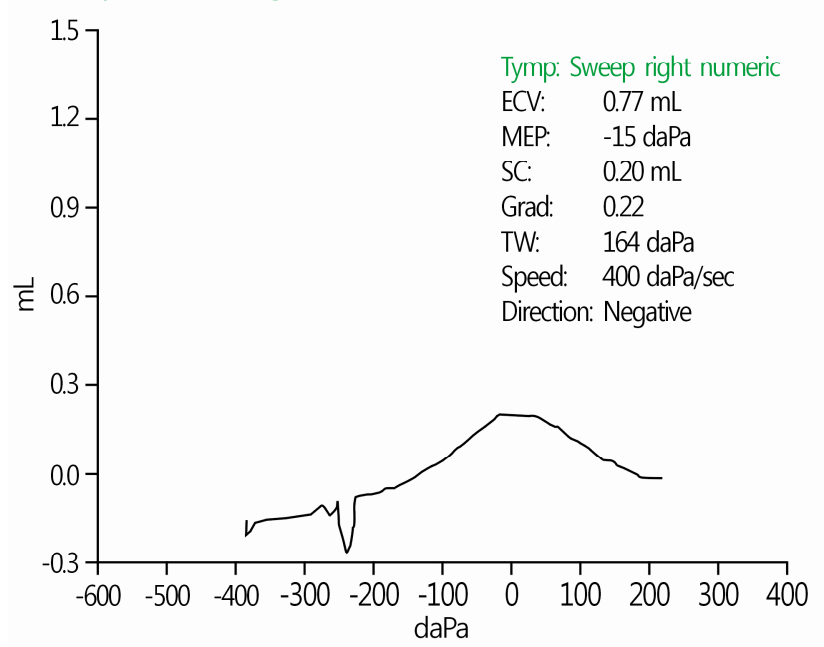

squamous epithelium and keratin debris, and the diagnosis of cholesteatoma was confirmed (Fig. 5)

\section{DISCUSSION}

Congenital cholesteatoma located within the tympanic membrane is referred to as congenital ITMC. ITMC was first reported by Hinton in 1863, and is very uncommon [4,11]. The exact pathogenesis, natural history, and prognosis are still unknown [12]. Sobol et al. suggested that the most attractive explanation for the development of ITMC is the basal cell papillary proliferation theory. This theory assumes that recurrent inflammation may stimulate basal cell proliferation, which can cause the formation of cholesteatoma [7]. This explanation is consistent with our observations in the present case.

The development of an ITMC can cause several complications, such as ossicular involvement and subsequent impairment of hearing conduction, similar to any other cholesteatoma of the middle ear [3]. To avoid middle ear involvement, early diagnosis and the prevention of rupture of the cholesteatoma into the middle ear are required [13]. Therefore, patients with a congenital ITMC are treated with surgery [3-10].

The tympanic membrane is composed of three layers: an inner mucosal layer, a middle fibrous layer, and an outer epithelial layer [14]. In many reported cases, the middle layer of the tympanic membrane was intact after removal of the cholesteatoma [12]. This means that the middle ear was not involved, even after the operation. Thus, a transcanal approach

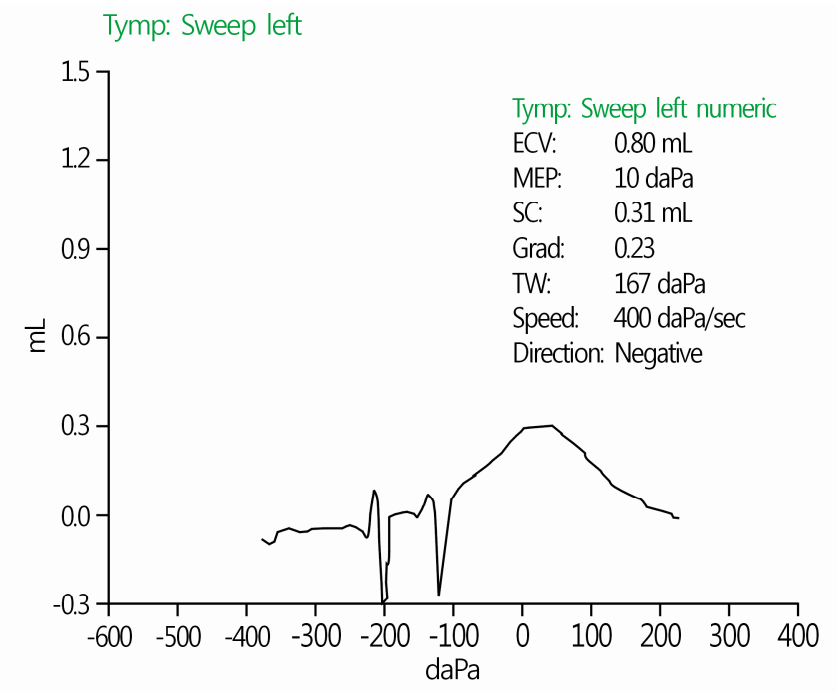

Fig. 2. Tympanograms. Tympanometry showed type A pattern in both ears. 

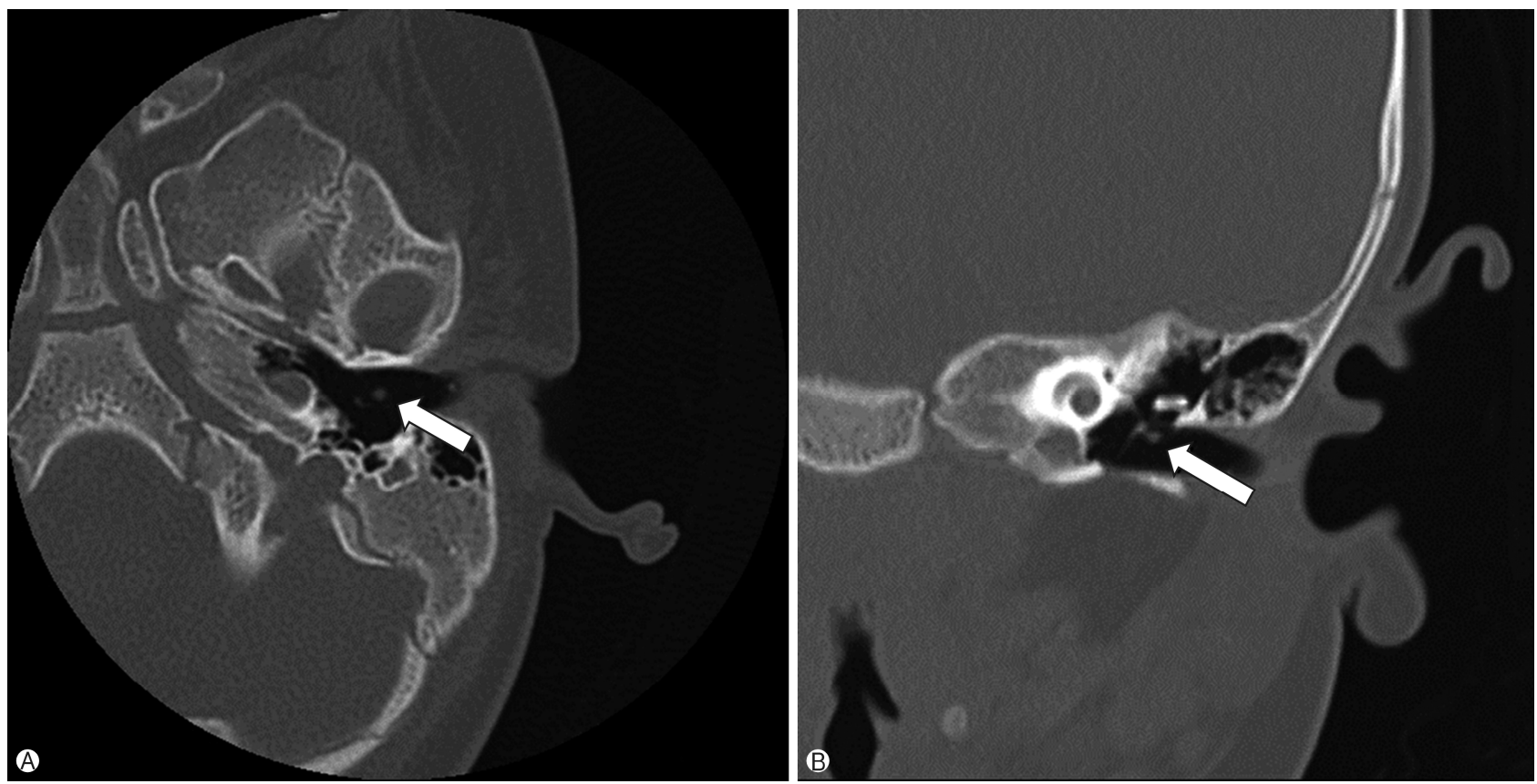

Fig. 3. Temporal computed tomography (CT). (A) Axial non-enhanced CT shows a soft tissue density of a round mass between the left tympanic membrane and the handle of the left malleus (arrow). (B) The soft tissue density is located at the lateral side of the handle of the left malleus (arrow).

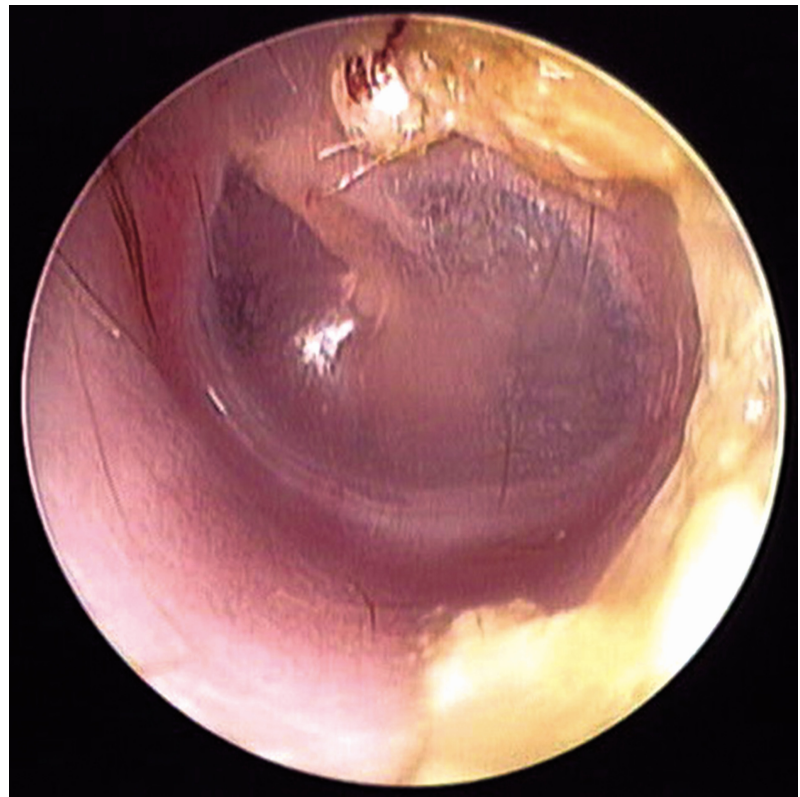

Fig. 4. Otoscopic finding after 2 months. The mass initially presumed to be an intratympanic membrane congenital cholesteatoma had moved laterally and became an external auditory canal mass.

is recommended for the removal of localized cholesteatoma, if the cholesteatoma can be peeled off from the tympanic membrane while maintaining an intact fibrous layer of the tym-

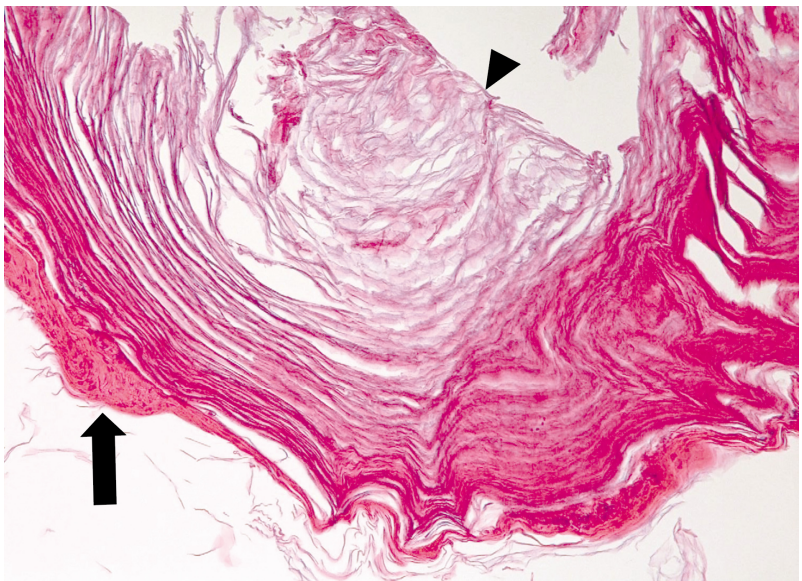

Fig. 5. Microscopic finding shows stratified squamous epithelium (arrow) and keratin debris (arrowhead) (H\&E stain, $\times 200)$.

panic membrane [3].

On the other hand, there are several reported cases of congenital ITMCs that changed into external auditory canal cholesteatomas on follow-up consultation [4,12]. Suzuki et al. [13] suggested that an ITMC can heal spontaneously when the cholesteatoma of the early stage ruptures into the auditory canal and keratin debris is completely removed. In this case, an ITMC moved laterally and became an external auditory canal mass within 2 months and was removed easily. 
Although the natural course of ITMC is not well known, most ITMCs likely increase in size over time [3].

Therefore, careful otomicroscopic examination and proper management are needed, even in pediatric patients who do not have any ear symptoms, to avoid progression beyond the tympanic membrane.

\section{CONFLICT OF INTEREST}

No potential conflict of interest relevant to this article were reported.

\section{ORCID}

Taehoon Kim, https://orcid.org/0000-0002-5796-6098

Da Jung Jung, https://orcid.org/0000-0001-6178-6113

\section{REFERENCES}

1. Levenson MJ, Michaels L, Parisier SC. Congenital cholesteatomas of the middle ear in children: origin and management. Otolaryngol Clin North Am 1989;22:941-54.

2. Olszewska E, Wagner M, Bernal-Sprekelsen M, Ebmeyer J, Dazert S, Hildmann H, et al. Etiopathogenesis of cholesteatoma. Eur Arch Otorhinolaryngol 2004;261:6-24.

3. Yoshida T, Sone M, Mizuno T, Nakashima T. Intratympanic membrane congenital cholesteatoma. Int J Pediatr Otorhinolaryngol 2009;73:1003-5.
4. Kim $\mathrm{YH}$. A case of intratympanic membrane congenital cholesteatoma. Korean J Otorhinolaryngol-Head Neck Surg 2012; 55:188-90.

5. Reddy CE, Goodyear P, Ghosh S, Lesser T. Intratympanic membrane cholesteatoma: a rare incidental finding. Eur Arch Otorhinolaryngol 2006;263:1061-4.

6. Smith R, Moran WB. Tympanic membrane keratoma (cholesteatoma) in children with no prior otologic surgery. Laryngoscope 1977;87:237-45.

7. Sobol SM, Reichert TJ, Faw KD, Stroud MH, Spector GJ, Ogura JH. Intramembranous and mesotympanic cholesteatomas associated with an intact tympanic membrane in children. Ann Otol Rhinol Laryngol 1980;89:312-7.

8. Weber PC, Adkins WY Jr. Congenital cholesteatomas in the tympanic membrane. Laryngoscope 1997;107:1181-4.

9. Pasanisi E, Bacciu A, Vincenti V, Bacciu S. Congenital cholesteatoma of the tympanic membrane. Int J Pediatr Otorhinolaryngol 2001;61:167-71.

10. Kim SA, Haupert MS. Congenital cholesteatoma of the tympanic membrane. Otolaryngol Head Neck Surg 2002;127: 359-60.

11. Hinton J. Sebaceous tumor within the tympanum originating on the external surface of the membrane tympani. Guy's Hosp Rep 1863;9:264

12. Jo MH, Yoo SW, Lim J, Lee SH. congenital intratympanic membrane cholesteatoma: report of three cases and review of the literature. Korean J Otorhinolaryngol-Head Neck Surg 2013;56:302-5.

13. Suzuki T, Nin F, Hasegawa T, Taki M, Sakaguchi H, Yamamoto $\mathrm{S}$, et al. Congenital cholesteatoma in the tympanic membrane. Int J Pediatr Otorhinolaryngol Extra 2007;2:48-50.

14. Donalson JA, Duckert LG. Anatomy of the ear. In: Paparella MM, Shumrick DA, Gluckman JL, Meyerhoff WL, editors. Otolaryngology. 3rd ed. Philadelphia: Saunders; 1991. p. 23-58. 